\title{
EL USO DEL DISCURSO REFERIDO EN EL CORPUS PRESEEA - SEVILLA: EL SOCIOLECTO ALTO ${ }^{1}$ THE REPORTED SPEECH USE IN THE PRESEEA CORPUS OF SEVILLE: THE HIGH SOCIOLECT
}

\author{
Doina Repede \\ Universidad de Sevilla \\ doinarepede@gmail.com \\ DOI
}

Resumen

El propósito de este artículo consiste en describir el uso del discurso reproducido en una muestra de habla compuesta por 24 entrevistas del sociolecto alto, pertenecientes al Corpus PRESEEA-Sevilla. Para ello, hemos trabajado con una plantilla de codificación formada por 8 variables independientes - lingüísticas, pragmáticas y sociales-. Los datos obtenidos muestran una preferencia por el uso del discurso directo por parte de los hablantes entrevistados. Además, este recurso permite graduar el tipo de cita atendiendo al nivel de compromiso con el discurso referido. El marco introductor preferido por estos informantes es mediante un verbo y decir es el más empleado para introducir las palabras referidas. Asimismo, según los factores sociales, son la primera generación y las mujeres quienes muestran mayor tendencia al uso del discurso reproducido.

Palabras Clave: discurso reproducido, sociolecto alto, Sociolingüística, corpus, PRESEEA-Sevilla

\section{Abstract}

The aim of this study is to describe the reported speech use in a sample of 24 oral interviews of high sociolect, taken from the Corpus PRESEEA- Seville; we based our study on a codification template formed by 8 independent variables -linguistic, pragmatic and social-. The results showed that the questioned informants preferred to use direct speech. Additionally, this mechanism allows sequencing the type of quotation according the level of involvement in their discourse. The most frequently introducing frame was with a verb and decir was the most employed verb to report. Also, the first generation and women showed a bigger tendency to the reported speech use.

\footnotetext{
${ }^{1}$ Quiero expresar mi agradecimiento a los dos revisores anónimos de Anuario de Letras. Lingüística y Filología, cuyos comentarios acertados han contribuido a una mejora de la versión definitiva de este estudio. Todos los errores y deficiencias que puedan encontrarse son únicamente de mi responsabilidad.
} 
KEYwORDS: reported speech, high sociolect, Sociolinguistic, corpus, PRESEEA-Seville

FECHA DE RECEPCIÓN: 18/07/2017

FECHA DE ACEPTACIÓN: 27/10/2017

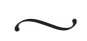

\section{Introducción ${ }^{2}$}

El discurso referido o reproducido "se pone de manifiesto en aquellas secuencias textuales que son el resultado de un acto de enunciación de carácter citativo" (Gallucci, 2016: 201). Mediante este recurso, el hablante cita palabras propias o ajenas en un momento distinto al que fueron realizadas originalmente. Es "específico del lenguaje humano" (2016: 201) y en todas las lenguas, ${ }^{3}$ "es un fenómeno lingüístico distinto a la producción original del mismo" (Maldonado, 1999: 3551).

El español presenta varios mecanismos para referir actos comunicativos, $y$, entre ellos, se distinguen, como los más utilizados, el discurso directo (DD) y el discurso indirecto (DI). El discurso directo se define como un procedimiento "mediante el cual quien habla (o escribe) incorpora a su discurso una secuencia textual, de procedencia propia o ajena, que es presentada como si supuestamente fuera reproducida de modo literal" (San Martín y Guerrero, 2013: 260). A diferencia del lenguaje escrito donde el segmento reproducido aparece marcado por signos gráficos, en el lenguaje oral son los indicios prosódicos, o el uso de un verbo introductor de discurso, cuando aparece, los que señalan el tipo de acto de habla atribuido a la cita (2013: 260):

\footnotetext{
${ }^{2}$ Este trabajo forma parte del proyecto Patrones Sociolingüísticos del Español de Sevilla (PASOS-SE). Proyecto de Excelencia convocatoria 2015. Ref. FFI2015- 61871C. Financiado por el Ministerio de Economía y Competitividad de España y Fondo Europeo de Desarrollo Regional (MINECO/FEDER, UE).

${ }^{3}$ En este caso se podría hablar de una perspectiva tipológica del discurso referido, ya que las lenguas cuentan con distintos tipos de mecanismos para referir el discurso original.
} 
(1) y cuando mi padre se enteró que vivía en La Alameda decía $<\mathrm{cita} />$ ipero cómo le digo yo a mis amigos que mi hija vive en La Alameda / por Dios! <cita/> (SEVI_M33_071). ${ }^{4}$

El discurso indirecto se caracteriza por el empleo de la conjunción que y consiste en la reproducción de lo dicho "desde el sistema de referencias deícticas del hablante que reproduce (tiempo de la subordinada, pronombres, ciertos adverbios, etc.)" (Maldonado, 1999: 3551). En el mismo orden de ideas, la RAE y ASALE (2009: 3273) señalan que mediante el discurso indirecto se reproducen las palabras de otro adaptándolas al sistema de referencias deícticas del hablante:

(2) y no me pude ni poner de pie / vamos / <vacilación/> completamente sentado / me atendieron / y parecía <vacilación/> parecía que podía ser algo de menisco / me quisieron operar y mi padre dijo que de ninguna manera / que yo no me iba a dedicar nunca al fútbol / profesionalmente / y que / por tanto / de tocarme la rodilla ni hablar(SEVI_H33_058).

Sin embargo, la conversión de este discurso al directo impide "construir un discurso original a partir del discurso indirecto, pues la operación de paráfrasis es de absoluta responsabilidad del locutor-reproductor y afecta a toda la cita, elecciones léxicas, adaptación de referencias deícticas, etc." (Méndez, 2000: 151-152).

Cabe mencionar que, aunque no se trata de un discurso indirecto convencional, hemos optado en este estudio por considerar como discurso indirecto ejemplos de tipo (3):

(3) $\mathrm{y}<$ alargamiento/> me vine / y aquí ya me contó Miguel Ángel el enorme riesgo que es un <alargamiento/> mosquito de esos (SEVI_H33_058). ${ }^{5}$

\footnotetext{
${ }^{4}$ Los ejemplos deben leerse de la siguiente forma: i) SEVI=Sevilla; ii) género: $\mathrm{H}=$ hombre, $M=$ mujer; iii) edad: $1=20$ a 34 años, $2=35$ a 54 años, $3=55$ años en adelante; iv) grado de instrucción: 3 = más de 13 años de escolaridad; v) identificación del hablante dentro del corpus: 049, 050, 051, etc.

${ }^{5}$ En estos ejemplos no aparece el nexo que y la subordinada del discurso indirecto estándar corresponde a un sintagma nominal.
} 
Como podemos ver, estamos ante una reformulación total del contenido del discurso reproducido, donde el locutor actual alude de manera expresa a un acto comunicativo de otro interlocutor, realizado en una situación de enunciación diferente a la actual (Repede, 2017b). Esta reproducción está acompañada por un verbo, en nuestro caso contar, que "transmite de modo más o menos transparente la intención comunicativa" (San Martín, 2015: 85). Se diferencia, así, del discurso narrativizado estándar, según se muestra en el siguiente fragmento:

(4) el profesor invitado lógicamente fue B / y B decidió que yo tenía que ir a ese viaje // yo lo propuse en casa $\mathrm{y}<$ alargamiento/> me apoyaron <alargamiento/> va < palabra_cortada/ $>$ total y absolutamente y allá que fui / y estando en Grecia // ese año / sesenta y $<$ alargamiento/> siete (SEVI_M33_070). ${ }^{6}$

que se presenta como un modo de reproducción de discurso o pensamiento del hablante original, y en el que no puede percibirse ningún tipo de citación o reproducción del texto original (RAE y ASALE, 2009), y donde el verbo de comunicación es el elemento que sintetiza o condensa las palabras referidas (Repede, 2017b).

Asimismo, el discurso reproducido ha sido relacionado fundamentalmente con el estudio de los textos escritos, tanto literarios (Girón Alconchel, 1989; Ruano San Segundo, 2015, etc.) como periodísticos (Casado Velarde, 2010; Casado Velarde y de Lucas Vicente, 2013; Méndez, 1999, 2000; Repede, 2015a; 2015b; 2017 a; etc.). Sin embargo, desde hace ya un tiempo se ha venido estudiando los tipos de discurso en la oralidad y su uso en la interacción conversacional. Por tanto, las investigaciones de Camargo (2008), Van der Houwen (2000), Gallucci (2008, 2013), Gallucci y Vargas (2015), Fernández (2012), San Martín y Guerrero (2013), San Martín (2015), Repede (2017b), etc. describen el empleo del discurso directo e indirecto en la lengua oral.

${ }^{6}$ Este ejemplo ilustra los casos que hemos descartado del corpus. 
El objetivo general de esta investigación consiste en describir y analizar el empleo del DD y DI en una muestra actual del habla de la comunidad urbana de Sevilla. En consonancia con lo anterior, nos proponemos, entre otros: a) indicar el marco introductor y los verbos de comunicación empleados por los hablantes para introducir DD y DI; b) identificar a quién atribuyen los sujetos los actos de habla que refieren; c) establecer las funciones pragmáticas del discurso reproducido; $\mathrm{y}$ d) determinar si el uso de estos mecanismos de discurso depende de los factores sociales considerados en la muestra de habla seleccionada. Asimismo, este análisis resulta pertinente por el hecho de poder aportar datos que reflejen el uso del discurso referido en esta ciudad, con el fin de que sean contrastados con los estudios hechos en las diferentes comunidades de habla hispana. ${ }^{7}$

\section{Metodología}

\subsection{Corpus y criterios de selección}

Para llevar a cabo nuestro análisis se han usado 24 entrevistas semidirigidas del sociolecto alto recogidas entre 2009 y 2015 y que forman parte del corpus PRESEEA en la ciudad. Cada una de las grabaciones tiene una duración de aproximadamente 45 minutos. Como criterios extralingüísticos, se han tenido en cuenta los siguientes:

a) Género. La división entre hombre y mujer "is critical in any research on variation and change, due to differences regarding linguistic behaviour, prestige and leadership" (Lastra y Martín Butragueño, 2015: 9).

b) Edad. Nuestro estudio, según los parámetros establecidos por PRESEEA, distingue entre tres variantes: i) de 20 a 34 años; ii) de 35 a 54 años; iii) de 55 en adelante. Esta división corresponde a "social divisions: accommodation in society; individual fullness

${ }^{7}$ Véase bibliografía. 
and social establishment; individual maturity and retirement. Of course, age is the key to study variation and change in apparent time" (2015: 9).

En el cuadro 1, se especifica la distribución de los informantes en el corpus utilizado para este análisis:

\begin{tabular}{lrcc}
\hline Edad & $\begin{array}{c}1^{a} \text { generación } \\
(\mathbf{2 0 - 3 4} \text { años })\end{array}$ & $\begin{array}{c}2^{a} \text { generación } \\
(\mathbf{3 5 - 5 4} \text { años })\end{array}$ & $\begin{array}{c}3^{a} \text { generación } \\
\text { (55 años o más) }\end{array}$ \\
\hline Hombro & 4 & 4 & 4 \\
Mujer & 4 & 4 & 4 \\
\hline
\end{tabular}

Cuadro 1: Distribución del sociolecto alto en el corpus PRESEEA-Sevilla

Para que las muestras utilizadas sean equivalentes y comparables, hemos analizado 30 minutos de cada grabación, esto es, hemos seleccionado todos los casos de discurso reproducido del minuto 5 hasta el 35 de cada entrevista. Hemos tomado como unidad de análisis el enunciado, que se describe como una estructura formada por una o varias oraciones o por alguna unidad menor (Gallucci y Vargas, 2015). ${ }^{8}$ Se trata de actos de habla que transmiten un mensaje, con una intención, según se refleja en los fragmentos (5) y (6):

(5) cuando yo entra < palabra_cortada/ $>$ entré <alargamiento/ $>$ / pues mira / me ech < palabra_cortada/ $>$ me $<$ alargamiento/ $>$ / nada más entrar los primeros días dije $<$ cita/ $>$ ¡uf! $<$ cita/ $>/$ una fatiga // el olor // a mí me <vacilación/> me impactó / nada más entrar / el olor / la primera semana (SEVI_M13_061).

(6) hay semanas que / mmm como <vacilación/> puedo comer en $<$ alargamiento/> en donde yo trabajo / o <alargamiento/> porque hay comidas / que claro / si hay en <vacilación/> en mi casa comida / pues digo <cita/> mamá voy a comer aquí / porque es que se echa a perder <cita/> (SEVI_M13_061).

${ }^{8}$ Véase también Gallucci (2013). 
Asimismo, del total de secuencias $(\mathrm{N}=424)$ registradas, hemos descartado de nuestro estudio los siguientes casos:

a) el contenido reproducido es ininteligible o incompleto (3): ${ }^{9}$

(7) nada nada / me encanta probar todo / me encanta probar todo / yo en México recuerdo / que comí una cosa y digo ¿qué he comido? estaba buenísimo / o<alargamiento/ $>$ eh <vacilación/ $>$ un sabor especial / me dijeron $<$ cita $>$ es $<$ ininteligible/ $></$ cita $>$ (SEVI_M33_072).

(8) te digo que me lo pensé < vacilación/> me lo pensé y no sé por qué en esos momentos te decides y al fin y al cabo el tiempo dice <cita/> pues quizás hubiese hecho <secuencia_incompleta/> $<$ cita/> (SEVI_M23_067).

b) el verbo de decir funciona como marcador discursivo (9):

(9) yo ya no porque ya trabajo / pero yo he vivido la Semana Santa muchísimo / muchísimo / además ya te digo / de <vacilación/> de balcones / y de conocernos los sitios <alargamiento/> clave / y de quedar / y era / hacerte un itinerario / y recorrer (SEVI_ M23_067).

c) el verbo de decir presenta un valor realizativo (7). Aparece en presente de indicativo, en primera persona y no indica "la descripción de una acción sino su realización” (Galluci y Vargas, 2015: 81):

(10) el año pasado por ejemplo mi chica que se cayó / pues me llamaron pues con toda la confianza $<$ cita/ $>$ tranquilízate $<$ cita/ $>$ que $<$ vacilación $/><$ ruido = "chasquido boca" $/>$ que no te digo que no llamen a cualquier madre / las llamarán ¿no? / pero te ves con

\footnotetext{
${ }^{9}$ Se ha incluido entre paréntesis el número de casos encontrados y eliminados del análisis.
} 
esa confianza que sabes que <vacilación/> que son amigas ¿no? (SEVI_M23_067).

d) el verbo de decir aparece con un complemento predicativo (3). Su significado corresponde al del verbo llamar o apodar:

(11) mi tía vivía en un <vacilación/> en un pueblo / que era un $<$ vacilación/ $>$ / no era un pueblo / era un $<$ vacilación/ $>$ una aldea de un pueblo / de San Antonio / pero donde vivía ella era como una montaña / de hecho le decíamos la Heidi / en vez de tita Mercedes era la Heidi (SEVI_M23_067).

e) el contenido del discurso reproducido no aparece expresado de forma explícita (12):

(12) I: una situación muy difícil / yo tenía el año pasado un alumno / muy / listo inteligente / brillante / pero no le daba la gana / de hacer absolutamente nada

E: uhum

I: hablé con los padres / le mandé una notita / el alumno tuvo la deferencia de entregarla / cosa que no hacen siempre (SEVI_M33_072).

Estamos, como podemos ver, ante un caso de discurso narrativizado estándar que supone un locutor primario que emitió un discurso, pero aparece totalmente integrado en el locutor actual. Se trata, así, del grado cero del discurso reproducido (Perret, 1994).

\subsection{Criterios de análisis y procesamiento}

Hemos considerado para este análisis la variable dependiente del discurso referido (que se compone de las variantes DD y DI) y como variables explicativas las siguientes: ${ }^{10}$

Variables lingüísticas: 1. Marco introductor de cita. 2. Tipo de verbo introductor de cita. 3. Presencia/ausencia de la conjunción que; 4. Duplicación de discurso referido.

Variables pragmáticas: 5 . Tipos de cita; 6 . Función de la cita.

\footnotetext{
${ }^{10}$ Utilizamos en este estudio el modelo propuesto para el análisis del sociolecto bajo en la comunidad de habla de Sevilla (Repede, 2017b).
} 
Variables sociales: 7. Género del hablante, 8. Edad del hablante.

En cuanto a los criterios sintácticos, nos centramos, en primer lugar, en el marco introductor de las citas en DD y DI, que presenta las siguientes variantes:

a) con verbo introductor (conjugado o no):

(13) entonces llamé a Miguel Ángel Y. y le dije <cita/> oye / que aquí hay una epidemia de dengue $<$ cita/ $/$ y me dijo $<$ cita/ $>$ coge el primer avión que haya para España $<$ cita/ $>$ / digo $<$ cita/ $>$ bueno / M. Á. / que yo tengo un contrato todavía / que me quedan aquí diez o doce días <cita/> (SEVI_H33_058).

b) sin verbo introductor:

(14) ahora / también tengo que decirte que hay ambientes / y situaciones / en las que uno comprende y / <cita/> hija / mira dame $<\mathrm{cita} />$ / según la tienda que sea / <cita/> ay / mira hija ¿por qué no me echas una mano? ¿por qué no me das? <cita/> / ¿eh? (SEVI_M33_070).

c) con un sintagma nominal precedido o no por la conjunción $y$ :

(15) I: mis últimas Navidades / mi hermana y yo pedimos <alargamiento/ $>$ a los Reyes unos legos $<$ risas= "I" $/>$ E: esto ya con treinta años ¿no? $<$ risas $=$ "E”/ $>$ I: con treinta años / que mi padre el pobre se quedó como diciendo $/$ mira $<$ risas= "E" $/>/$ y yo $<$ cita/ $>$ papá que sí / que nos hace mucha ilusión $<$ cita/ $>$ / y $<$ alargamiento/ $>$ / porque los legos son carísimos (SEVI_M23_066).

d) a través de un marcador del discurso:

(16) y los padres vinieron a hablar conmigo desesperados / porque $<$ cita/> es que no sabemos qué hacer con él <cita/> / y como esos hay muchos / muchos padres y sus hijos o se les rebelan o se queda $<$ alargamiento/ $>$ n sin hacer nada / o deciden no hacer nada (SEVI_M33_072). 
e) otros. Esta variante incluye diferentes estructuras nominales seguidas de la preposición de, según se refleja en los ejemplos (17) y (18):

(17) pienso que < alargamiento/> el andaluz < vacilación/> la gente / no lo considera aceptable pero / porque el andaluz tiene muchos tópicos de <alargamiento/> <cita/> los del Sur son catetos / hablan en andaluz / no hablan bien <cita/> ¿no? (SEVI_M23_065).

(18) pero/ también/ que sea por un problema que tú estés tan gordo que tu salud<ininteligible/> tu salud le perjudica / entonces me parece bien hacer dieta // pero si tú comes bien y haces deporte $<$ alargamiento/ $>0$ andas $0<$ alargamiento/ $>0$ estás bien / o te sientes bien contigo mismo // no tiene $<$ vacilación/ $>$ lo que pasa es que si hay mucha presión social de <cita/> ¡ay / que gorda estás! / ¡no estoy en el pantalón! / ¡ay / en esa tienda no me puedo comprar! / ¡ay / pues no tengo dinero! <cita/> (SEVI_M23_065).

En segundo lugar, consideramos los verbos introductores de las citas. Como es obvio, nos centramos únicamente en aquellos casos en los que se utilice un verbo como introductor de discurso reproducido. Esta variable se desglosa en dos variantes:

a) el verbo decir como introductor de discurso directo:

(19) abuela C. dijo <cita/> vamos a regalarle a Lucía un Nenuco $<\mathrm{cita} />$ / yo dije <cita/> ¿para qué? / que luego <alargamiento/> / que no va a querer <cita/ $>$ / que tal / y <alargamiento/ $>$ incluso estuve diciéndole un par de semanas antes de Reyes a Lucía $<$ cita/> Lucía a lo mejor los Reyes te traen < alargamiento/> un muñeco $<$ cita/> (SEVI_M23_066).

b) otro verbo, distinto del verbo decir, como referente de discurso reproducido:

(20) la directora / nos exige <cita/> venga / excursiones / hacer cosas diferentes <cita/> vale / pero es que / los únicos que se mueven / 
o que < alargamiento/> es que no < vacilación/ > no < vacilación/ $>$ / no nos < alargamiento/ $>$ facilitan nada / ni autobuses ni / furgonetas adaptadas / hombre si la mayoría está en carrito de ruedas / ¿cómo nos desplazamos? (SEVI_M13_061).

Otro criterio sintáctico es la duplicación del discurso reproducido por medio de un pronombre o un adverbio. ${ }^{11}$ Esta variable presenta tres variantes. Las dos primeras sirven para codificar las formas en que se produce la duplicación del discurso referido (catafórica, en los ejemplos (21) y (22), y anafórica, según el fragmento (23)):

(21) sí sí pero él le encantan mmm y hace así: <cita/> mmm abuelito iqué rico! <cita/> / sí sí </simultáneo> o sea que me defiendo en la cocina pero no puedo decir que sea un gran cocinero no (SEVI_H33_059).

(22) yo tenía una amiga allí en Santander que además me lo decía / que <vacilación/> que ella pensaba que en el carácter influenciaba mucho lo que era el clima / y puede ser / ellos son quizá más cerrados / más introvertidos / no sé si será el clima más frío (SEVI_M23_067).

(23) yo cuando me < vacilación/ $>$ me <alargamiento/> / me admitieron en el máster / yo fui / y dije / mira / esto es dos opciones / o yo / me hago autónoma / y vengo a trabajar tres <vacilación/>

${ }^{11}$ Como ya se ha demostrado en estudios anteriores (Repede, 2015a; 2015b), el discurso reproducido no siempre es complemento directo, sino que puede desempeñar el papel sintáctico de complemento circunstancial. En este caso, es posible la duplicación del discurso referido mediante un adverbio:

"eso no sirve para nada / si yo lo que quiero es trabajar / entonces ellos / además directamente me lo decían así <cita/> yo lo que quiero es trabajar $<$ cita/> (SEVI_M13_062).

Se ve claramente en este ejemplo que la cita no actúa como objeto directo. El pronombre lo remite a un contenido anterior del discurso y el adverbio así hace referencia a la cita propiamente dicha. 
tres semanas al mes / o < vacilación/ > o me tengo que ir / y ellos me dijeron que fuera mi padre / sin cobrar esa semana

E: a sustituirte

I: a sustituirme / y así fue cuatro años / y eso no lo propuse / me lo < vacilación/> me lo propusieron ellos y así se hizo / y bueno mi padre allí ha trabajado y ha hecho muchas cosas gratis también (SEVI_M13_063).

La tercera variante acoge los casos en los que no hay repetición:

(24) hay semanas que / mmm como <vacilación/> puedo comer en $<$ alargamiento/> en donde yo trabajo / o <alargamiento/> porque hay comidas / que claro / si hay en <vacilación/> en mi casa comida / pues digo <cita/> mamá voy a comer aquí / porque es que se echa a perder <cita/> (SEVI_M13_061).

En cuanto al aspecto sintáctico "presencia/ausencia de la conjunción que", esta variable se desglosa en dos variantes. La primera se refiere a los casos en los que el discurso referido (tanto el directo como el indirecto) aparece con la conjunción que:

(25) siempre preguntamos / eh <alargamiento/> ¿te ha servido? / ite ha servido esto de algo? / o yo siempre suelo preguntar en terapia ¿te < vacilación/>te está sirviendo? / ¿te < vacilación/ $>$ te está aportando esto algo positivo? / ¿estás igual? ¿estás? <vacilación/> / y casi siempre / $\mathrm{mmm} /$ la respuesta es que <cita/> bueno / que le está sirviendo <cita/> (SEVI_M13_062).

La segunda variante incluye las muestras en las que el discurso referido se utiliza sin la conjunción que, como se refleja en la siguiente muestra:

(26) una vez que me jubile / todavía me quedan unos años / pero $<$ risas=I/ $>$ / yo no lo espero cuando me jubile / lo espero cada día / porque creo que la vida hay que vivirla cada día / entonces cuando a mí me dicen <cita/> pero tú ¿por qué no descansas? por 
la tarde / estás tranquilo <cita/> pues porque para mí / parte de la vida es moverme / y necesito moverme (SEVI_H33_057).

En cuanto a los factores pragmáticos, hemos tomado en consideración las siguientes variables: tipo de cita y función de la cita. ${ }^{12}$ La primera permite conocer a quién atribuyen los hablantes los actos de habla reproducidos y el nivel de su implicación o compromiso en la cita (Gallucci y Vargas, 2015). Así, esta variable se desglosa en cuatro variantes:

a) cita propia. El hablante reproduce sus propias palabras expresadas en otro momento del discurso y se compromete más con lo que dice:

(27) ya que hacía escala allí / digo <cita/> pues me quedo aquí unos dos o tres días $<$ cita/> (SEVI_M33_072).

b) cita ajena. El sujeto refiere la palabra de otro y se compromete menos discursivamente:

(28) cuando mi padre se enteró que vivía en la Alameda decía <cita/> ipero cómo le digo yo a mis amigos que mi hija vive en la Alameda! $<$ cita/> (SEVI_M33_071).

c) cita impersonal. Las palabras referidas no remiten ni al propio hablante ni al referente ajeno, sino que el discurso referido se emplea con un uso impersonal:

(29) entonces llega un momento que tú te tienes que frenar y decir $<$ cita/> mira vamos a ver / que yo no quiero problemas / que yo lo que quiero es disfrutar de esto / que no nos vamos a enfadar $<$ cita/> (SEVI_M23_067).

d) cita encubierta. El hablante resguarda su responsabilidad sobre lo dicho y señala que la información procede de terceros:

\footnotetext{
${ }^{12}$ Véase también Fernández (2012); Gallucci y Vargas (2015), Repede (2017b).
} 
(30) yo creo que en cuanto la gente se lleva aquí un tiempo y se acostumbra a oír hablar / entienden lo que se dice porque no es $<$ vacilación/> no son palabras raras ni nada / es un acento / en principio ¿no?/ que luego hay cosas que aquí se llaman de una forma y allí de otra / vale / vamos / puede ser ¿no? / que aprendas dos o tres palabras / pero el problema que dice la gente es que no nos entiende porque hablamos muy rápido y nos comemos palabras I entonces / bueno / eso es como todo / te acostumbras a oírlo y al final lo entiendes ¿no? // dicen que <vacilación/> que no hablamos bien (SEVI_H23_055).

Según la intención del hablante que emplea este recurso y la secuencia discursiva de la que forma parte, la cita puede presentar distintas funciones (Camargo, 2008; Gallucci, 2009; Fernández, 2012):

a) relatar (una anécdota, experiencias personales o ajenas):

(31) yo estaba mmm pues cuidando de los gatitos / le estaba dando de comer y tal / entonces una tarántula me empezó a subir por la espalda / y mi marido me dio un golpe / me volví y le dije <cita/> ipero qué haces! <cita/> / y me dice <cita/> no / nada / que tenías una cosa ahí <cita/> / entonces no le dio ninguna importancia pero yo me extrañé porque me dio bastante fuerte / y pensé <cita/> pero cómo me da este golpe tan fuerte <cita/> (SEVI_M23_068).

b) ejemplificar: $:^{13}$

(32) por ejemplo una chica me dijo la semana pasada $<$ cita/ $>$ pues yo estuve ya con <alargamiento/> diez años con una psicóloga y nunca me hizo nada / y esto me está haciendo mucho más de lo que yo esperaba $<$ cita/> (SEVI_M13_062).

\footnotetext{
${ }^{13}$ Aunque los límites entre la función de relatar y ejemplificar son difusos y, en general, pueden superponerse, se ha considerado como función de ejemplificar, cuando "la cita forma parte de una secuencia narrativa y sirve para ilustrar una circunstancia" (Gallucci y Vargas, 2015: 80).
} 
c) expresar pensamientos:

(33) E: ¿y Estados Unidos lo conoces?

I: no // bueno estuve / cuando estuve en México / eh / paré porque era más barato hacer el vuelo por Miami y estuve en Miami tres días me parece o cuatro días / ya que <alargamiento/> E: jah! claro

I: ya que hacía escala allí digo <cita/> pues me quedo aquí unos dos o tres días <cita/> es lo que conozco de Estados Unidos (SEVI_M33_072).

d) argumentar:

(34) no hay una / $\mathrm{mmm} /$ responsabilidad muy grande cuando < vacilación/> / bueno / no somos muy responsables / en el sentido de $<$ alargamiento/> laboral / ¿no? / que a lo mejor en otros países sí he visto que / si dicen <cita/> mañana hay que tener este trabajo $<\mathrm{cita} />$ se tiene ese trabajo / aquí / bueno / es más / menos un día / el < vacilación/ > el típico < vacilación/ > la típica frase ¡ya nos vemos! / pues no es ya nos vemos mmm de verdad / sino que $<$ alargamiento/> puede que ocurra o puede que no (SEVI_M13_064).

Cabe mencionar aquí que las encuestas han sido procesadas mediante el programa Sketch Engine, ${ }^{14}$ una herramienta diseñada para el estudio de datos lingüísticos y filológicos. Asimismo, para ver si las variables utilizadas en este análisis han resultado significativas para el uso de los mecanismos de discurso referido en el sociolecto alto de la ciudad de Sevilla, hemos empleado la prueba del $\mathrm{x}^{2}$, que nos permite determinar si las frecuencias observadas en el cruce de variables tienen significación estadística o no. Para ello, los resultados de las variables no deben superar el límite de $0.05{ }^{15}$

\footnotetext{
${ }^{14}$ http://www.sketchengine.co.uk/

${ }^{15}$ Para los casos con menos de cinco ejemplos, hemos aplicado también la prueba exacta de Fisher.
} 


\section{Estudio y resultados del uso del discurso referido en el corpus}

En las 24 entrevistas pertenecientes al sociolecto alto y que forman parte del Corpus Sociolingüístico del proyecto PRESEEA en Sevilla, hemos podido identificar 390 secuencias de discurso reproducido. En la gráfica 1 se presentan las frecuencias de empleo de las dos variantes dependientes del discurso referido registradas en nuestros materiales, esto es, discurso directo (DD) y discurso indirecto (DI):

Gráfica 1. Frecuencia absoluta y relativa de DD y DI

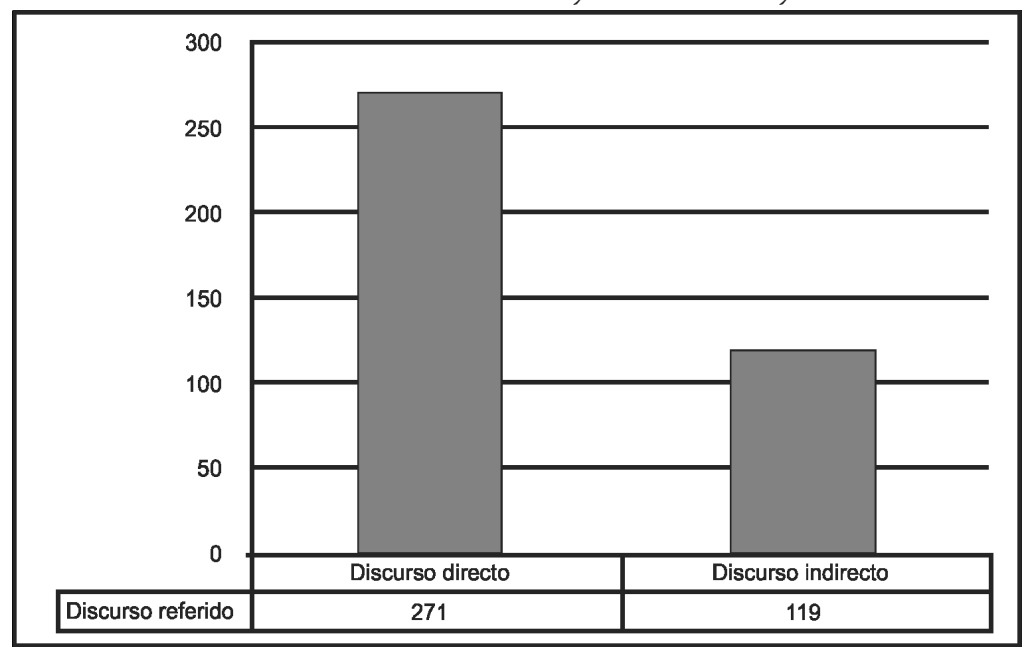

Como podemos observar, la distribución de las formas de discurso referido muestra un reparto desigual entre el uso de DD y el DI en el corpus manejado: se ha registrado, así, una mayor frecuencia de DD correspondiente a 271 casos $(69.31 \%)$, a diferencia del DI con 119 secuencias (30.69\%) del total de la muestra. Estos resultados coinciden con los obtenidos en el trabajo sobre el sociolecto bajo (Repede, 2017b) donde también el DD es más frecuente que el DI: se ha documentado un total de 595 secuencias, donde 400 ocurrencias $(67.23 \%)$ corresponden al DD y 195 secuencias (32.77\%) al DI. 


\subsection{Uso de discurso referido según factores sintácticos}

En la gráfica 2 podemos observar que del total de formas de discurso reproducido $(\mathrm{N}=390)$ encontradas en los materiales utilizados, 346 muestras aparecen con verbo como marco introductor, esto es, $88.71 \%$, donde 227 ejemplos pertenecen al DD y 119 casos al DI. El siguiente marco más utilizado es [-verbo] con 35 casos, todos de DD, lo que supone un $8.97 \%$.

Gráfica 2. Discurso reproducido y marco introductor

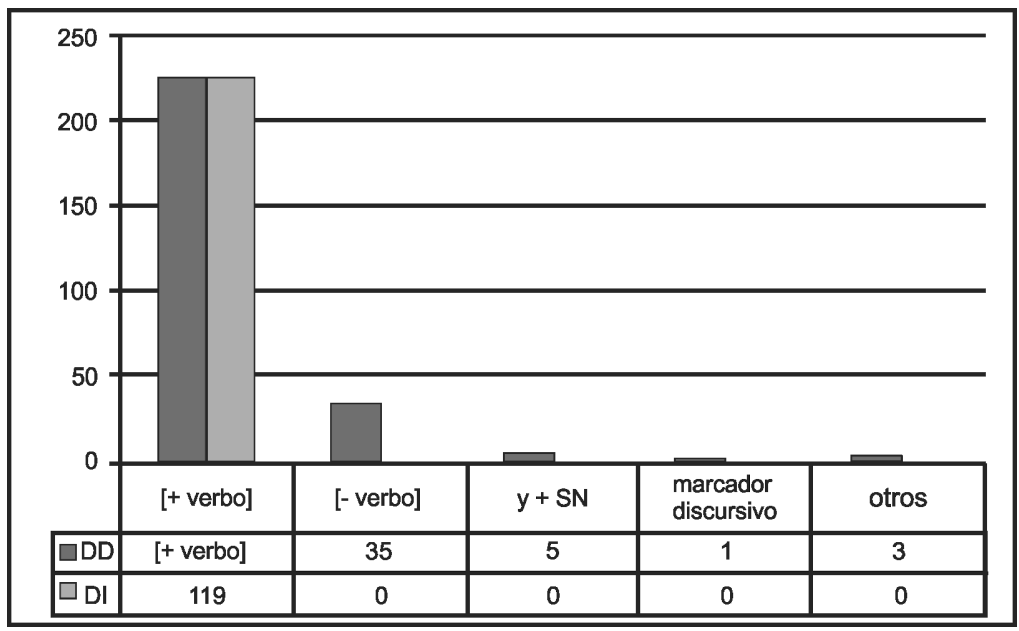

$$
x^{2}=21,778>9,488 \text { g.d.l. } 4 p=0,000<0,05^{16}
$$

Cuando el marco introductor está representado por la construcción $y+S N$, hemos identificado 5 ejemplos (1.28\%). Si se trata de un marcador del discurso, se ha encontrado solo 1 muestra $(0.26 \%)$. En cuanto a la variante otros, se han localizado 3 ejemplos, los que supone un $0.77 \%$. Asimismo, según se refleja en la gráfica, se trata únicamente de secuencias con DD.

Aunque no hemos contemplado ningún parámetro prosódico, cabe mencionar que para identificar este tipo de secuen-

\footnotetext{
${ }^{16} \mathrm{Al}$ aplicar la prueba de Fisher, el valor de $p$ es igual a 0,0001 , por tanto la variable resulta significativa desde el punto de vista estadístico.
} 
cias nos hemos basado en la entonación. Así, a diferencia de los actos de habla introducidos por un verbo, en estos casos, es la prosodia la que señala el discurso reproducido e indica, por tanto, la voz que se cita. Se trata de un cambio de melodía, ritmo y pausa que produce un contraste relativo al segmento previo (Sams, 2007).

Otra variable que ha resultado explicativa es el tipo de verbo introductor de discurso con sus dos variantes: el verbo decir y otro verbo. En la gráfica 3 presentamos, de los 347 casos de la muestra introducidos por un verbo, la proporción entre el verbo decir y los otros verbos:

Gráfica 3. Discurso reproducido y tipo de verbo introductor

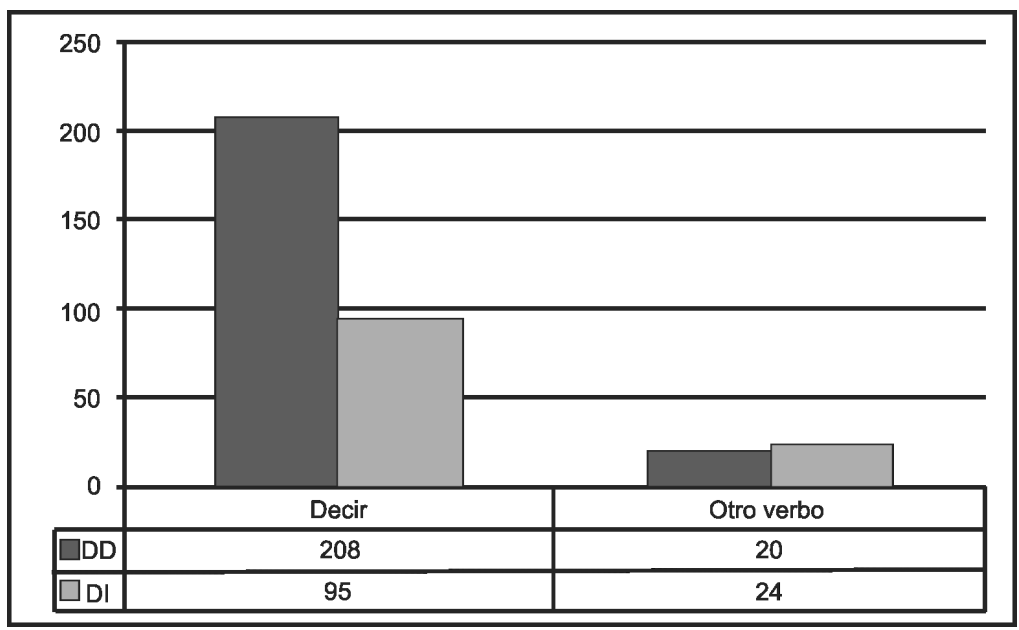

$$
\mathrm{x}^{2}=9,171>3,841 \text { g.d.1. } 1 p=0,0024<0,05
$$

En este análisis, el verbo más empleado para introducir tanto DD como DI es decir con un total de 303 casos (87.31\%), donde 208 ejemplos pertenecen al DD y 95 al DI. Aunque en la oralidad el inventario de verbos no es tan variado como puede serlo en los textos escritos, hemos encontrado 44 casos introducidos por otros verbos, lo que se traduce en un $12.68 \%$, nada desdeñable. De estos verbos, 20 introducen DD y 24 aparecen con DI. 
Así, hemos localizado en la muestra utilizada para este estudio verbos de:

a) comunicación como advertir (1), ${ }^{17}$ contar (3), comentar (1), confesar (1), contestar (1), enseñar (1), explicar (2), hablar (1), insistir (2), plantear (1), preguntar (8), proponer (1), repetir (1), subrayar (1),

b) petición: exigir (1) y pedir (3).

c) elección: decidir (2);

d) cognición: pensar (5);

e) otros verbos y locuciones verbales. Es el caso de seguir (1), ser (3), engatusar (1), hacer (1), publicar (1) y tener claro (1):

(35) la bendición / claro / hombre esto pasa en el ámbito normal / y entonces / eh <alargamiento/ $>$ / en el hombre como atormentado de todo ese tiempo / el < vacilación/ $>$ manda decir que por favor / que <alargamiento/ $>$ avisen a $<$ alargamiento/ $>$ / a esta señora / se llamaba Isolina / entonces / eh / que avisen a esta señora / porque <alargamiento/ $>\mathrm{mmm}$ quería reconocerle / a $<$ alargamiento/> la hija que tuvo / con él / y fueron a la aldea / estaba como a unos <alargamiento/> / kilómetro y medio más o menos / a por ella / y la contestación de ella fue <cita/> eh $<$ alargamiento/> / no recuerdo haber tenido ningún hijo con este señor < cita/> (SEVI_H33_057).

(36) sí sí pero él le encantan mmm y hace así <cita/> mmm abuelito ¡qué rico! <cita/> / sí sí </simultáneo> o sea que me defiendo en la cocina pero no puedo decir que sea un gran cocinero no (SEVI_H33_059).

El caso de los verbos ser o hacer es muy interesante, porque, como podemos ver, es el contexto el que los convierte en introductores de discurso referido. Si bien el verbo ser del ejemplo /35/ se aleja de la noción de "decir", en el contexto aparece ligado

\footnotetext{
${ }^{17}$ Entre paréntesis hemos incluido para cada verbo el número de casos encontrados en el corpus.
} 
a "la contestación", de modo que puede introducir la cita. En el ejemplo /36/, es posible que el hablante acompañe la cita de gestos. En este caso, el verbo hacer enfatiza el gesto y se omite decir, resultando, así, compatible con la noción de discurso.

Otro de los criterios sintácticos que ha resultado significativo es la duplicación del discurso referido. La circunstancia no duplicación es la más frecuente en la muestra manejada, según se refleja en la siguiente gráfica:

\section{Gráfica 4. Discurso reproducido y duplicación}

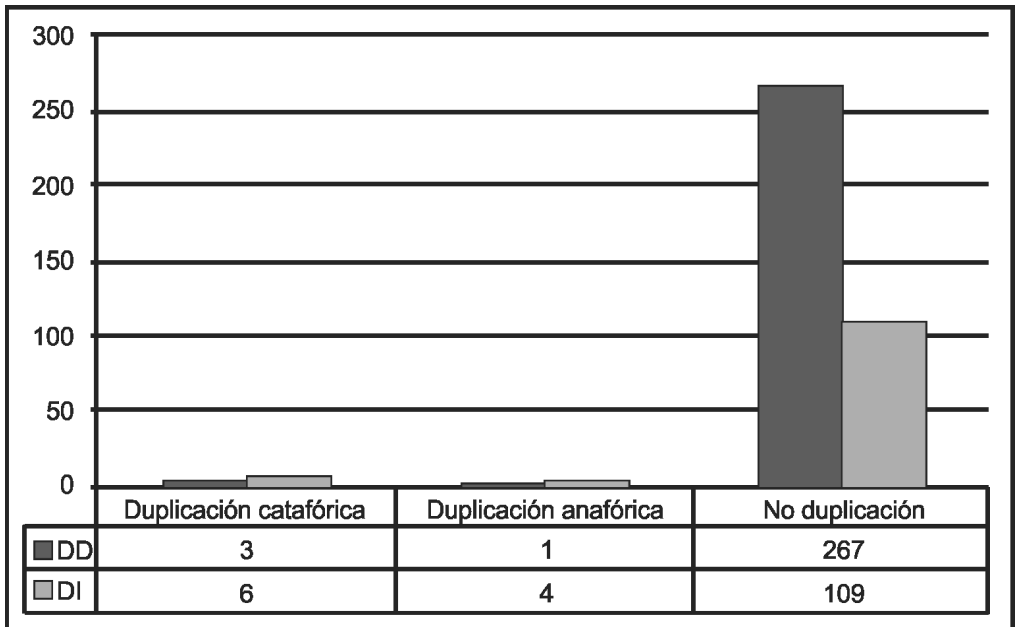

$$
\mathrm{x}^{2}=11,735>5,991 \text { g.d.l. } 2 p=0,003<0,05^{18}
$$

Aparece, así, en 376 casos $(\mathrm{N}=390)$, lo que significa un 96.41\%, donde 267 son muestras de DD y 109 de DI.

Con respecto a la duplicación, se produce un contexto menos favorable para las variantes de discurso referido. Se han documentado 9 ejemplos (2.31\%) con duplicación catafórica, esto es, 3 ejemplos de DD y 6 de DI. Asimismo, hemos encontrado solo 5 muestras para la duplicación anafórica, lo que se traduce en un $1.28 \%$. Se trata de 1 caso de DD y 4 de DI. ${ }^{19}$

\footnotetext{
${ }^{18}$ El valor de $p$ según la prueba exacta de Fisher es igual a 0,003.

19 Señalamos que las ocurrencias de DI con duplicación anafórica implican la reiteración del verbo de habla en el enunciado, ya que esta estructura impide la 
El criterio sintáctico "presencia/ausencia de la conjunción que" también ha resultado estadísticamente significativo. Cabe mencionar aquí que nos hemos centrado únicamente en los casos de discurso directo e indirecto estándar, esto es, en aquellas muestras donde es posible el uso de esa conjunción. ${ }^{20}$ Por tanto, para esta variable se han documentado 312 casos, según se refleja en la gráfica 5:

Gráfica 5. Discurso reproducido y presencia/ausencia de que

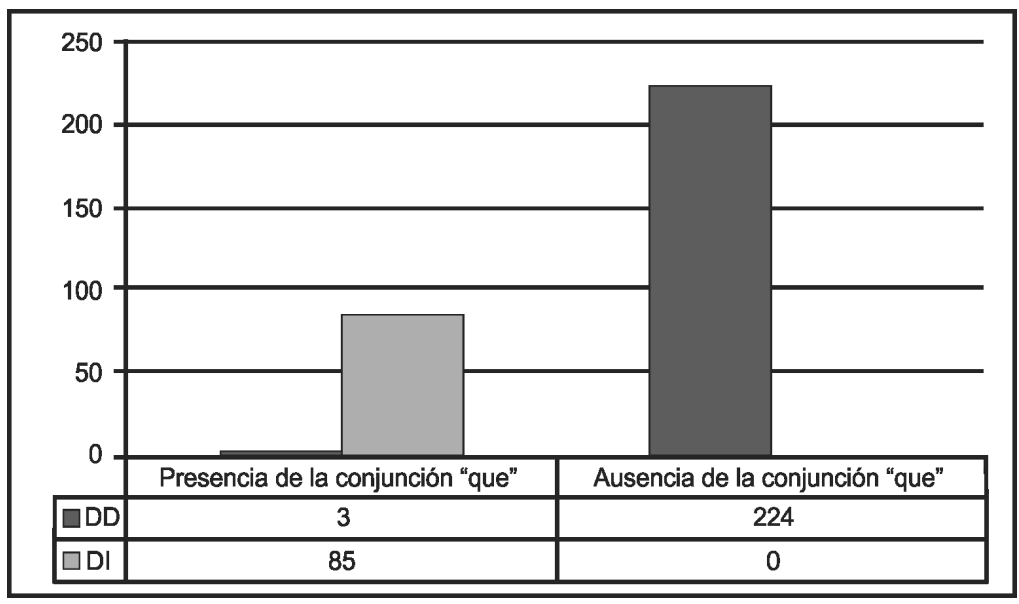

$$
x^{2}=293,688>3,841 \text { g.d.l. } 1 p=0,0001<0,05^{21}
$$

Según nuestro corpus, los informantes entrevistados, al momento de reproducir una cita, eliden la conjunción en 224 casos (71.79\%), a diferencia de los 88 ejemplos introducidos por la conjunción que (28.21\%).

Si desglosamos por tipo de discurso, observamos que el DD aparece sin la conjunción que en 224 casos (98.68\%), a diferencia de las 3 muestras registradas con este nexo (1.32\%). Cuando

aparición de un pronombre o adverbio que haga referencia a la cita reproducida y que aparezca después de dicha cita, sin que se repita el verbo de comunicación.

${ }^{20}$ Se han eliminado las ocurrencias de discurso directo libre, los casos de discurso referido con un marco introductor distinto al de un verbo y estructuras de tipo "y aquí me contó M.A. el enorme riesgo que es un mosquito de esos” (SEVI_H33_058), porque generalmente se utilizan sin la conjunción que.

${ }^{21}$ El valor de $p$ en la prueba exacta de Fisher es igual a 0,0001. 
los hablantes reproducen en DI se observa la tendencia opuesta: emplean la conjunción en 85 casos (100\%) encontrados en la muestra, frente a ninguna ocurrencia sin el que.

\subsection{Uso de discurso referido según factores pragmáticos}

Aparte de los criterios sintácticos descritos hasta ahora, es necesario también "conocer a quién(es) los hablantes le atribuyen la palabra citada, pues esto refleja en qué medida se comprometen con lo que dicen" (Gallucci y Vargas, 2015: 88). Como ya se ha mencionado, esta variable presenta cuatro variantes: cita propia, cita ajena, cita impersonal y cita encubierta.

En la gráfica 6 podemos observar que de los 390 casos estudiados, la cita propia se encuentra en 135 ejemplos (34.62\%) y la cita ajena aparece en 154 secuencias, que representan un $39.49 \%$ del total. En cuanto a las últimas dos variantes, hemos documentado 51 ocurrencias (13.08\%) para la cita impersonal y 50 muestras $(12.82 \%)$ para la cita encubierta. Según estos datos, podemos observar que la diferencia entre los dos últimos parámetros es de apenas 0.26 puntos porcentuales. Asimismo, estos resultados permiten concluir que, al menos en la muestra analizada, en líneas generales, es más frecuente la cita ajena.

Gráfica 6. Discurso reproducido y tipo de cita

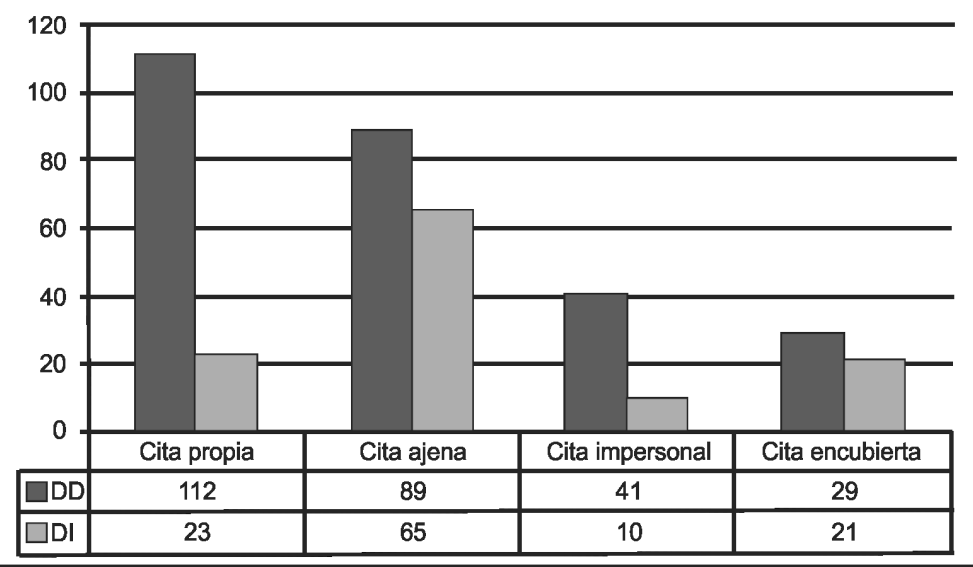

$\mathrm{x}^{2}=27,469>7,815$ g.d.1. $3 p=0,000<0,05$ 
Según Chafe (1982), el DD se presenta como uno de los rasgos que caracteriza el coloquio. Así, como podemos observar en nuestra gráfica, el DD predomina en todos los tipos de cita. En concreto, en la cita propia $(\mathrm{N}=135)$, notamos que el DD muestra una inclinación bastante alta hacia la reproducción de palabras propias (82.96\%), en detrimento del DI (17.04\%). Con respecto a la cita ajena $(\mathrm{N}=154)$, el DD se utiliza en 89 casos $(57.79 \%)$ y el DI en 65 ejemplos (42.21\%). Cuando se trata de la cita impersonal ( $\mathrm{N}=51)$, el DD aparece 41 veces $(80.39 \%)$, a diferencia de los 10 ejemplos (19.61\%) de DI. Para la cita encubierta $(\mathrm{N}=50)$, hemos encontrado 29 casos (58\%) de DD y 21 ejemplos (42\%) de DI.

Asimismo, uno de los rasgos que daría cuenta de la implicación (Chafe, 1982) 22 en el discurso oral es el DD, y que, como hemos visto, es el que se emplea con mayor frecuencia en los materiales utilizados. El DI, por su parte, se vincula con el distanciamiento. Sin embargo, consideramos que según el grado de responsabilidad o compromiso del hablante con las palabras referidas, podríamos hablar de un continuum dentro del DD según el tipo de cita, ${ }^{23}$ así como aparece reflejado en la gráfica 7.

Esta escala va desde un mayor nivel de implicación con las palabras reproducidas, que determina el uso de la cita propia, hasta un menor nivel de compromiso con respecto al acto de habla referido, característico del empleo de la cita encubierta.

Gráfico 7: Escala de DD según el tipo de cita

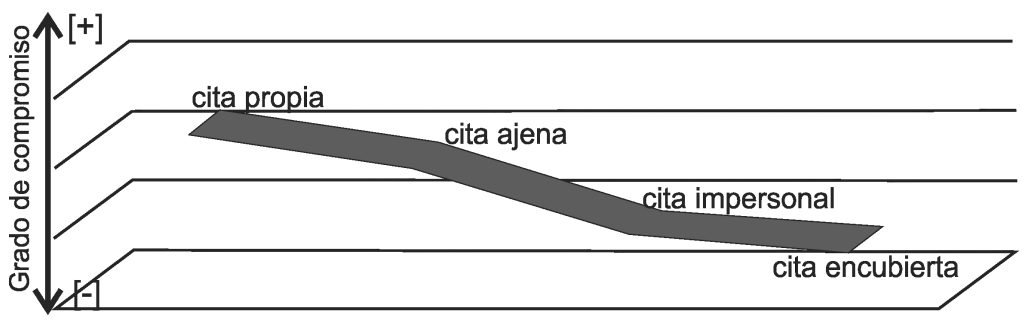

\footnotetext{
${ }^{22}$ Este autor utiliza los conceptos de compromiso (involvement) y distanciamiento (detachment) para referirse a los rasgos lingüísticos que diferencian el lenguaje oral del lenguaje escrito.

${ }^{23}$ Véase también el estudio sobre el sociolecto bajo (Repede, 2017b) en el corpus PRESEEA-Sevilla.
} 
Así, el hablante puede involucrarse en lo que dice y citar sus propias palabras, o, por el contrario, recurrir a la cita encubierta para desmarcarse de la responsabilidad de las palabras reproducidas.

En la gráfica 8 presentamos la distribución de las funciones de la cita, según la intención del hablante que emplea este recurso y de la secuencia discursiva de la que forma parte: relatar (una anécdota, experiencias personales o ajenas), ejemplificar, expresar pensamientos y argumentar.

Gráfica 8. Discurso reproducido y función de la cita

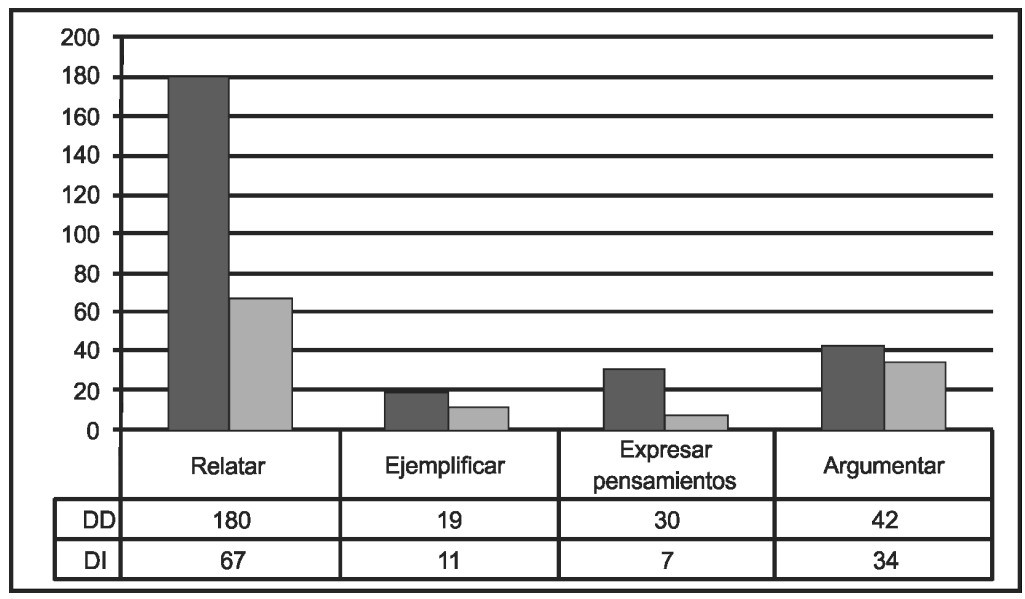

$$
\mathrm{x}^{2}=11,47>7,815 \text { g.d.l. } 3 p=0,009<0,05
$$

De acuerdo con los datos proporcionados en esta gráfica, tanto el DD como el DI se registran mayoritariamente en las secuencias discursivas de tipo narrativo, aunque con una evidente predominancia del DD. En concreto, la función más frecuente es la de relatar anécdotas o experiencias con 247 casos (63.33\%) de los 390 de la muestra utilizada para este estudio. Le sigue, en orden descendente, la función de argumentar, correspondiente a 76 ejemplos (19.49\%), manifestar un pensamiento, con 37 ocurrencias $(9.49 \%)$, y, por último, la circunstancia de ejemplificar, con 30 casos $(7.69 \%)$.

Si desglosamos por tipo de discurso, el DD se emplea en todas las funciones de la cita mencionadas: relatar con $180 \mathrm{ca}-$ 
sos (66.42\%), ejemplificar con 19 ejemplos (7.01\%), expresar un pensamiento con 30 ocurrencias (11.07\%) y argumentar con 42 casos (15.5\%). Por lo que se refiere al comportamiento del DI, se ha manifestado en las secuencias narrativas con 67 ocurrencias (56.30\%), en las argumentativas con 34 casos (28.57\%), y, en menor proporción, en la función de ejemplificar con 11 muestras (9.24\%), y, en la de expresar pensamientos, donde se han localizado solo 7 secuencias $(5.88 \%)$.

\subsection{Uso del discurso referido según factores sociales}

En relación con el género de los informantes encuestados, la gráfica 9 muestra los resultados obtenidos según los dos tipos de uso contemplados en este estudio:

Gráfica 9. Discurso referido y la variable género

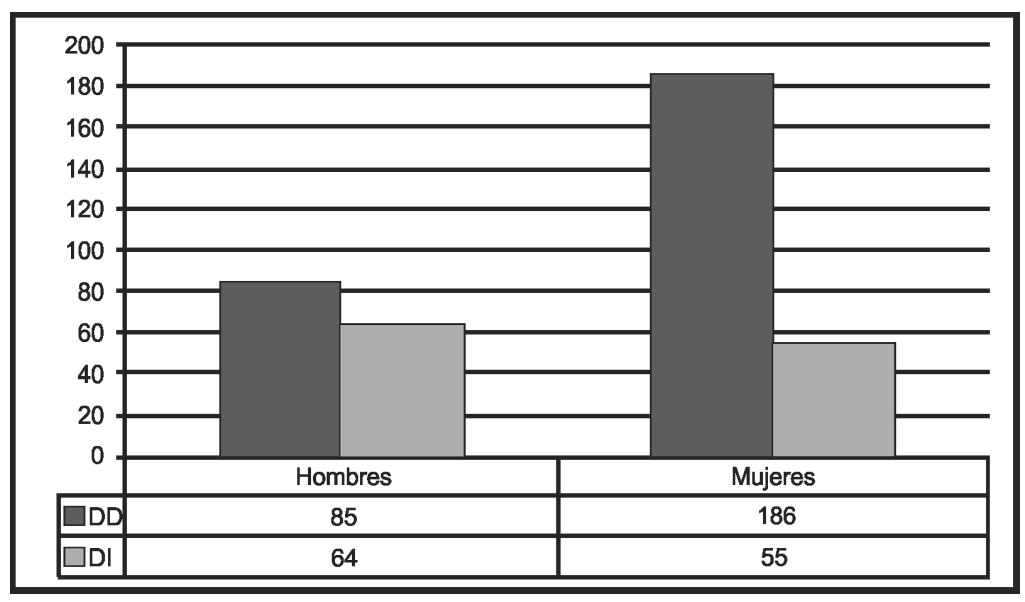

$$
\mathrm{x}^{2}=17,6>3,841 \text { g.d.l. } 1 p=0,000<0,05
$$

Podemos observar que de los 390 casos de discurso reproducido, 149 ejemplos (38.21\%) pertenecen a los hombres (85 de DD y 64 de DI). Por su parte, las mujeres citan más, esto es, 241 (61.79\%) secuencias de discurso referido, donde 186 pertenecen al DD y 55 al DI. Así, puede apreciarse que, según nuestra mues- 
tra, las mujeres del sociolecto alto emplean con más frecuencia los procedimientos de cita y muestran $23.58 \%$ puntos porcentuales de diferencia con respecto al uso del discurso referido que realizan los hombres.

Si nos fijamos solo en el DD utilizado como recurso para citar, del total de 271 secuencias, los hombres utilizan este mecanismo en un $31.37 \%$, y las mujeres un $68.63 \%$. En este caso, hablamos de una diferencia de 30.26 puntos porcentuales, que se traduce en unas frecuencias de empleo de discurso directo muy lejanas entre hombres y mujeres. En cambio, la diferencia es menor para el DI, ya que del total de 119 ejemplos, los hombres recurren a este procedimiento un $53.78 \%$ y las mujeres un $46.21 \%$, es decir, se trata de 7.57 puntos porcentuales.

Cabe mencionar aquí que en el estudio hecho sobre el nivel bajo (Repede, 2017b) encontramos también que las mujeres citan más que los hombres (56.97\% vs. 43.03\%). Así, estos resultados se ven corroborados por los obtenidos en otras investigaciones sobre el tema que nos ocupa: en San Martín y Guerrero (2013: 272) sobre el habla de Santiago de Chile, el empleo del discurso referido se manifiesta "preferentemente en las mujeres, aunque se trata de frecuencias de aparición muy cercanas a las registradas en las entrevistas de los hombres que conforman la muestra"; en Gallucci (2008) sobre el habla de Caracas, las mujeres citan más que los hombres (55\% vs. $45 \%$ ) o en Fernández (2012) donde el 60\% de los casos del habla de Mérida corresponden a citas de mujeres.

Sin embargo, afirmar que las mujeres citan más que los hombres parece ser "una tesis difícil de mantener, ya que las tendencias no se reflejan de la misma manera en las distintas investigaciones" (Gallucci y Vargas, 2015: 91). Por ejemplo, en Gallucci (2009) sobre el habla de Caracas, los hombres citan en un $75 \%$ de las muestras de su corpus.

En la siguiente gráfica se reflejan los resultados obtenidos de acuerdo con la variable edad: 
Gráfica 10. Discurso referido y la variable edad

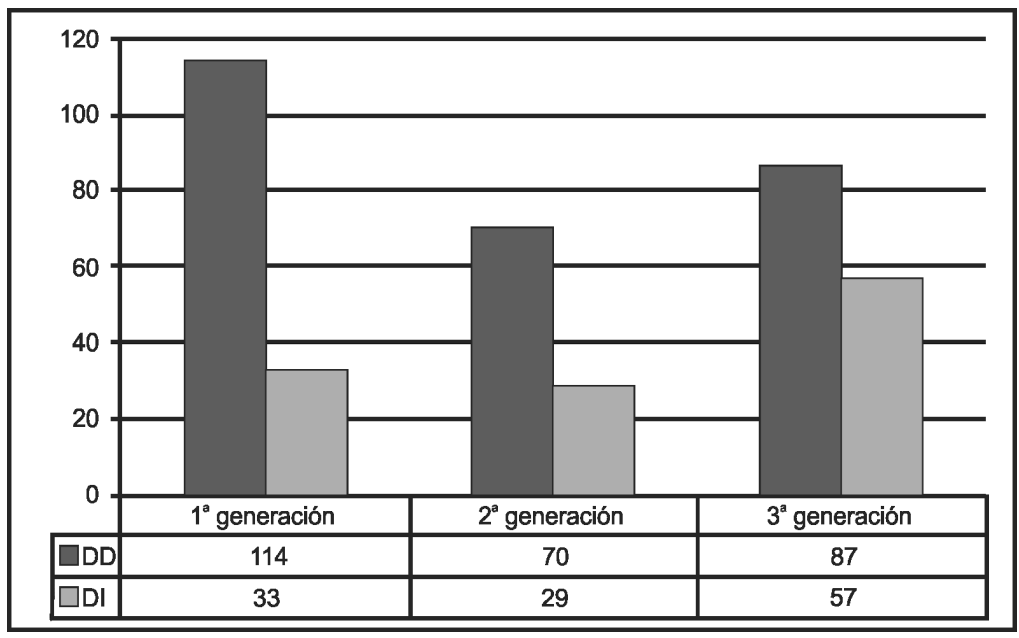

$$
x^{2}=10,166>5,991 \text { g.d.1. } 2 p=0,0062<0,05
$$

Como se desprende de los datos de la gráfica 10, es el primer grupo generacional el que más uso de este recurso hace, con 147 ocurrencias (37.69\%), seguido por el grupo de tercera generación, con 144 casos (36.93\%), de modo que el empleo del discurso reproducido se produce de manera casi similar entre el primer y el tercer grupo etario. Sin embargo, la frecuencia de empleo del discurso referido disminuye levemente para el grupo de mediana edad, que registra 99 casos, esto es, $25.38 \%$.

Se puede apreciar, así, que del total de casos $(\mathrm{N}=390)$, el $\mathrm{DD}$ es el que se utiliza con mayor frecuencia en los tres niveles, aunque con una mayor tendencia en el primer grupo etario, con el 29.23\%, en tanto que su empleo disminuye en la segunda generación, con el $17.95 \%$, y sube otra vez en el tercer grupo, que registra un $22.31 \%$ de las ocurrencias.

El DI, por su parte, se mantiene relativamente constante en los dos primeros niveles, con un $8.46 \%$ y un $7.43 \%$, respectivamente, y aumenta su frecuencia en el tercer nivel, con el $14.62 \%$. Como consecuencia, la distancia entre el empleo del DD y el DI es mucho más ostensible en el grupo etario de 20 a 34 años.

No obstante, estos datos difieren de los que encontramos en el sociolecto bajo con respecto a esta variable. Para ese nivel 
hallamos que es el segundo grupo generacional el que más uso de este recurso hace (47.06\%), seguido por el grupo de tercera generación (31.09\%). En cuanto a los hablantes de primera generación, son los que menos citan (21.85\%).

\section{Conclusiones}

Los resultados obtenidos del análisis de 24 muestras de habla extraídas del Corpus Sociolingüístico del proyecto PRESEEA en Sevilla coinciden en su mayoría con los de otras investigaciones llevadas a cabo sobre el tema tratado (Gallucci, 2013; Fernández, 2012; Gallucci y Vargas, 2015; Repede, 2017b). Así pues, encontramos que el DD se emplea más que el DI (69.49\% vs. $30.51 \%)$.

Por factores sintácticos, observamos que el DD sigue siendo la forma más usada por los hablantes para citar y el discurso referido suele introducirse con un verbo que, en general, es decir. En cuanto al marco introductor, en este estudio, al igual que en el de Repede (2017b), se observa que los hablantes disponen de al menos cinco opciones para introducir el discurso referido en DD y DI. En ambas investigaciones los datos indican que estos prefieren introducir las citas, sobre todo, con un verbo, $y$, en menor medida, sin él. Asimismo, la circunstancia no duplicación es la más frecuente, y en relación con la presencia/ausencia de $q u e$, se utiliza el nexo para el DI y se elide cuando se reproduce en DD.

Por factores pragmáticos, los hablantes entrevistados prefieren citar el discurso de otros y suelen hacerlo empleando el DD. Este mecanismo permite graduar el tipo de cita atendiendo al nivel de responsabilidad con las palabras referidas: cita propia $>$ cita ajena > cita impersonal > cita encubierta. La secuencia discursiva más habitual en la que aparece el discurso referido es la de relatar una anécdota o experiencia personal y/o ajena seguida por la de argumentar.

En cuanto a las variables sociales consideradas, los resultados ponen de manifiesto una frecuencia bastante grande de em- 
pleo del discurso referido según el sexo de los informantes: se ha registrado un mayor porcentaje de aparición en las mujeres con un $61.79 \%$ de citas y frente al $38.21 \%$ de los hombres. Como ya se ha mencionado, también en el sociolecto bajo, encontramos que las mujeres citan más que los hombres.

En cambio, con respecto al grupo de edad, si en el nivel bajo encontramos que era la segunda generación quien más uso hacía de este mecanismo, en el sociolecto alto el discurso referido se manifiesta en el primer grupo etario un 37.69\% y un $36.93 \%$ en el tercer grupo, mientras que en el segundo grupo de edad la frecuencia de discurso referido baja (25.38\%).

A la vista de los resultados obtenidos, parece evidente la necesidad de futuros trabajos respecto del fenómeno estudiado a partir de una misma metodología, que nos permitirán comprobar la validez de estos hallazgos, así como observar la variación en la producción de recursos discursivos en otras variedades del español.

\section{Bibliografía}

Camargo Fernández, L. (2008), “La representación del discurso en los corpus PRESEEA: Metodología y propuesta de análisis", ponencia presentada en XV Congreso Internacional de la Asociación de Lingüística y Filología de América Latina, Montevideo.

Casado Velarde, M. (2010), "Algunas estrategias para la desautorización del discurso ajeno en la prensa", en C. Martínez Pasamar (ed.), Estrategias argumentativas en el discurso periodístico, Fráncfort, Peter Lang, pp. 69-85.

Casado Velarde, M. y Alberto de Lucas (2013), "La evaluación del discurso referido en la prensa española a través de los verbos introductores", Revista Signos, 46, pp. 332360. Disponible en <http://www.redalyc.org/articulo.oa? $\mathrm{id}=157028891003>$.

Chafe, W. (1982), "Integration and involvement in speaking, writing and oral literature", en D. Tannen (ed.), Spoken and 
written language. Exploring orality and literacy, Norwood, Ablex, pp. 35-53.

Fernández, M. (2012), "Discurso directo e indirecto en el español de Mérida”, Lengua y Habla, 16, pp. 71-85.

GAllucci, M. (2008), "Aproximación al uso del discurso directo e indirecto en el habla de Caracas", ponencia presentada en XV Congreso Internacional de la Asociación de Lingüística y Filología de América Latina, Montevideo.

Gallucci, M. (2009), "Nos fuimos a la casa y mi mamá: ‘ ¿estaban lanzándose por la quebrada?’. ‘no mamá!', 'claro que sí. Estudio piloto de las citas conversacionales en el habla de Caracas", Núcleo, 26, pp. 75-98.

GAllucci, M. (2013), "Más sobre el estilo directo e indirecto en el español de Caracas", Lengua y Habla, 17, pp. 89-111.

Gallucci, M. y K. Vargas (2015), "Estilo directo e indirecto en el Corpus Sociolingüístico de Caracas 1987", Anuario de Letras. Lingüística y Filología, III (2), pp. 65-103.

GALlucci, M. (2016), "El discurso referido en los manuales sobre análisis del discurso y pragmática lingüística", Lengua y Habla, 20, pp. 200-224.

Girón Alconchel, J. L. (1989), "Las formas del discurso referido en el 'Cantar de Mio Cid”', Boletín de la Real Academia Española, Anejo 44, Madrid.

HOUWEN, F. vAN DER (2000), "El habla directa vs. indirecta y la organización del discurso”, Foro Hispánico, 17, pp. 27-40.

Lastra, Y. y P. Martín Butragueño (2015), "Subject pronoun expression in oral Mexican Spanish", en A. M. Carvalho et al. (eds.), Subject pronoun expression in Spanish. A Cross-Dialectal Perspective, Washington, Georgetown University Press, pp. 39-57.

Maldonado, C. (1999), "Discurso directo y discurso indirecto", en I. Bosque y V. Demonte (dirs.), Gramática descriptiva de la lengua española, volumen 3, Madrid, Espasa, pp. 3551-3595. MÉndez, E. (1999), "Análisis de la reproducción del discurso ajeno en los textos periodísticos", en Pragmalingüística, 7, pp. 99-128. Disponible en línea en <http: // revistas.uca.es/ index.php/pragma/article/viewFile/502/436>. 
Méndez, E. (2000), “Análisis de las formas de introducir el discurso ajeno en los textos periodísticos: el contexto reproductor", en J. J. de Bustos Tovar (ed.), Lengua, discurso, texto, Madrid, Visor, pp. 2082-2098.

Perret, M. (1994), Lénonciation en grammaire du texte, París, Nathan.

PRESEEA-Sevilla (2009-2017), Proyecto para el Estudio Sociolingüístico del Español de España y América. Corpus Sociolingüístico del Español de Sevilla.

Real Academia Española y Asociación de Academias de la Lengua Española (2009), Nueva gramática de la lengua española, Madrid, Espasa Libros.

REPEDE, D. (2015a), "El discurso directo como estrategia de interpretación de la palabra ajena en los textos periodísticos", Lengua y Habla, 19, pp. 77-92.

Repede, D. (2015b), "Aspectos gramaticales y discursivos de los incisos de cita directa en los textos periodísticos", en A. de Lucas Vicente et al. (eds.), Aplicaciones y enfoques teóricos del análisis del discurso, Pamplona, EUNSA, pp. 95-117.

Repede, D. (2017a), "Los verbos atípicos introductores de discurso directo en los textos periodísticos: la interferencia del nivel discursivo", en A. M. Cestero Mancera y M. E. Olímpio (eds.), Investigaciones actuales en Lingüística. Vol. IV: Sobre el discurso, Alcalá de Henares, Universidad de Alcalá, Servicio de Publicaciones, pp. 17-34.

RePede, D. (2017b), "Análisis del discurso reproducido en el corpus PRESEEA-Sevilla: el sociolecto bajo", en J. M. Santos Rovira (ed.), Variación lingüística e identidad en el mundo hispanohablante, Lugo, Axac, pp. 55-74.

Ruano San Segundo, P. (2015), "Verbos de habla en textos literarios: propuesta metodológica para su localización y apuntes en torno a su función caracterizadora en Pickwick Papers, de Charles Dickens", en A. de Lucas Vicente et al. (eds.), Aplicaciones y enfoques teóricos del análisis del discurso, Pamplona, EUNSA, pp. 119-140. 
SAms, J. (2007), "Quoting the Unspoken: An analysis of quotations in spoken discourse", Colorado Research in Linguistics, 20, pp. 1-16.

San Martín, A. y S. Guerrero (2013), "Una aproximación sociolingüística al empleo del discurso referido en el corpus PRESEEA de Santiago de Chile", Revista Signos, 46 (82), pp. 258-282.

SAn Martín, A. (2015), Variación sintáctica y discursiva en el español hablado en Santiago de Chile, tesis doctoral, Valladolid, Universidad de Valladolid. Disponible en línea en <https:/uvadoc.uva.es/bitstream/10324/15865/1/Tesis746-160210.pdf $>$. 